\title{
Non-ohmic critical fluctuation conductivity of layered superconductors in magnetic field
}

\author{
I. Puice* and W. Lang \\ Institut für Materialphysik der Universität Wien, Boltzmanngasse 5, A-1090 Wien, Austria
}

\begin{abstract}
Thermal fluctuation conductivity for a layered superconductor in perpendicular magnetic field is treated in the frame of the self-consistent Hartree approximation for an arbitrarily strong in-plane electric field. The simultaneous application of the two fields results in a slightly stronger suppression of the superconducting fluctuations, compared to the case when the fields are applied individually.
\end{abstract}

PACS numbers: 74.20.De,74.25.Fy,74.40.+k

The high-temperature superconductors (HTSC) show a more pronounced effect of fluctuations in the normalsuperconducting transition region, due to their high critical temperature, small coherence length, and quasi-twodimensional nature. Outside the critical region above $T_{c}$, in the absence of magnetic field and for small electric fields, the fluctuation conductivity can be explained by the Aslamazov-Larkin 1 theory, subsequently extended by Lawrence and Doniach (LD) ${ }^{2}$ for two-dimensional layered superconductors, a situation very much resembling the crystal structure in cuprates.

The fluctuation conductivity may be however calculated in the linear-response approximation only for sufficiently weak electric fields, that do not perturb the fluctuation spectrum. ${ }^{3}$ Reasonably high values of the electric field accelerate the fluctuating paired carriers to the depairing current, and suppress the fluctuation lifetime, leading to deviation from the Ohm's law. In connection with the low-temperature superconductors, the nonlinearity has been studied theoretically for the isotropic case $\stackrel{4.5}{,}$ while more recently Varlamov and Reggiani ${ }^{6}$ and Mishonov et al $l^{\underline{7}}$ addressed the issue of the nonohmic fluctuation conductivity for a layered superconductor in an arbitrary electric field. The above mentioned theories describe however the fluctuations as noninteracting Gaussian ones, neglecting the quartic term in the Ginzburg-Landau (GL) free energy. This approximation holds not too close to the transition point, but it breaks down in the critical region, for higher densities of fluctuation Cooper pairs.

The Ohmic fluctuation conductivity in the presence of a magnetic field was also initially treated ${ }^{8.9}$ in the Gaussian fluctuation approach, which predicted a divergence at $T_{c}(H)$ that is however not observed. The physical reason is the motion of vortices providing dissipation and hence a finite flux-flow conductivity. Ikeda et al $\stackrel{10}{\underline{10}}$ and Ullah and Dorsey (UD) $\stackrel{11}{\Perp}$ showed that the theoretical divergence can be eliminated by using the Hartree approximation, which treats self-consistently the quartic term in the GL free-energy expansion. This approach was applied for the longitudinal ${ }^{10.11}$ and Hall conductivity, ${ }^{11}$ for the Nernst effect and the thermopower ${ }^{11}$ in the linearresponse approximation, for a layered superconductor under magnetic field.

In this paper we shall treat, in the self-consistent
Hartree approximation, the thermal fluctuation conductivity for a layered superconductor in a perpendicular magnetic field and for an arbitrarily strong in-plane electric field, a topic that, to our present knowledge, has not been treated yet. While the effect of the interacting fluctuations under applied magnetic field was investigated in the Hartree model ${ }^{10,11}$ only in the linear response approximation, that is for infinitesimally small electric fields, the non-linear conductivity for a layered system under arbitrarily strong electric field was derived in the Gaussian,,$\underline{6.7}$ as well as in the Hartree approximation, 12 but only in the absence of magnetic field.

For our purpose, we shall adopt the Langevin approach to the time-dependent Ginzburg-Landau (TDGL) equation ${ }^{4.11}$ The starting point of the formalism is the same as in Refs. 11 and 12, and consists of the LD expression of the GL free energy for a system of Josephson coupled superconducting planes, the gaugeinvariant relaxational TDGL equation governing the critical dynamics of the superconducting order parameter, and the Langevin white-noise forces that model the thermodynamical fluctuations, satisfying the fluctuationdissipation theorem. Keeping the same notations as in Ref. 12, the TDGL equation for the superconducting order parameter in the $l$-th plane will write

$$
\begin{array}{r}
\Gamma_{0}^{-1} \frac{\partial \psi_{l}}{\partial t}-i \frac{e_{0} \Gamma_{0}^{-1} E x}{\hbar} \psi_{l}+a \psi_{l}+b\left|\psi_{l}\right|^{2} \psi_{l} \\
-\frac{\hbar^{2}}{2 m}\left[\partial_{x}^{2}+\left(\partial_{y}-\frac{i e_{0}}{\hbar} x B\right)^{2}\right] \psi_{l} \\
+\frac{\hbar^{2}}{2 m_{c} s^{2}}\left(2 \psi_{l}-\psi_{l+1}-\psi_{l-1}\right)=\zeta_{l}(\mathbf{x}, t) .
\end{array}
$$

Here $m$ and $m_{c}$ are effective Cooper pair masses in the $a b$-plane and along the $c$-axis, respectively, $s$ is the distance between superconducting planes, and the pair electric charge is $e_{0}=2 e$. The order parameter has the same physical dimension as in the 3D case, and SI units are used. The perpendicular magnetic field $B$ is generated by the vector potential in the Landau gauge, $\mathbf{A}=(0, x B, 0)$, with $x$ and $y$ the in-plane coordinates, and the magnetization is neglected. The GL potential $a=a_{0} \varepsilon$ is parameterized by $a_{0}=\hbar^{2} / 2 m \xi_{0}^{2}=$ $\hbar^{2} / 2 m_{c} \xi_{0 c}^{2}$ and $\varepsilon=\ln \left(T / T_{0}\right)$, with $T_{0}$ the mean-field transition temperature, while $\xi_{0}$ and $\xi_{0 c}$ are, respec- 
tively, the in-plane and out-of-plane coherence lengths extrapolated at $T=0$. The relaxation rate $\Gamma_{0}$ in the TDGL equation is given by ${ }^{13} \Gamma_{0}^{-1}=\pi \hbar^{3} / 16 m \xi_{0}^{2} k_{B} T$. The Langevin white-noise forces $\zeta_{l}(\mathbf{x}, t)$ are correlated through $\left\langle\zeta_{l}(\mathbf{x}, t) \zeta_{l^{\prime}}^{*}\left(\mathbf{x}^{\prime}, t^{\prime}\right)\right\rangle=2 \Gamma_{0}^{-1} k_{B} T \delta\left(\mathbf{x}-\mathbf{x}^{\prime}\right) \delta(t-$ $\left.t^{\prime}\right) \delta_{l l^{\prime}} / s$, where $\delta\left(\mathbf{x}-\mathbf{x}^{\prime}\right)$ is the 2-dimensional deltafunction concerning the in-plane coordinates. The electric field $\mathbf{E}$ is assumed along the $x$-axis, generated by the scalar potential $\varphi=-E x$. In the chosen gauge, the current density operator along the $x$ direction in the $l$-th plane will give, after averaging with respect to the noise

$$
\left\langle j_{x}^{(l)}\right\rangle=-\left.\frac{i e_{0} \hbar}{2 m}\left(\partial_{x}-\partial_{x^{\prime}}\right)\left\langle\psi_{l}(\mathbf{x}, t) \psi_{l}^{*}\left(\mathbf{x}^{\prime}, t\right)\right\rangle\right|_{\mathbf{x}=\mathbf{x}^{\prime}} .
$$

As mentioned, the quartic term in the thermodynamical potential will be treated in the Hartree approximation, 11,14 by replacing the cubic term $b\left|\psi_{l}\right|^{2} \psi_{l}$ in Eq. (11) with $b\left\langle\left|\psi_{l}\right|^{2}\right\rangle \psi_{l}$. This results in a linear problem with a modified (renormalized) GL potential $\widetilde{a}=a+b\left\langle\left|\psi_{l}\right|^{2}\right\rangle$, and a renormalized reduced temperature

$$
\widetilde{\varepsilon}=\varepsilon+\frac{b}{a_{0}}\left\langle\left|\psi_{l}\right|^{2}\right\rangle
$$

We shall further introduce the Fourier transform with respect to the in-plane coordinate $y$, the layer index $l$, and time $t$, respectively, and also the Landau level (LL) representation with respect to the $x$-dependence, through the relation:

$$
\begin{aligned}
\psi_{l}(x, y, t) & =\int \frac{d k}{2 \pi} \int_{-\pi / s}^{\pi / s} \frac{d q}{2 \pi} \int \frac{d \omega}{2 \pi} \sum_{n \geq 0} \psi_{q}(n, k, \omega) \\
& \cdot e^{-i k y} e^{-i q n s} e^{-i \omega t} u_{n}\left(x+\frac{\hbar k}{e_{0} B}\right),
\end{aligned}
$$

where the functions $u_{n}(x)$ with $n \in \mathbb{N}$ build the orthonormal eigenfunction system of the harmonic oscillator hamiltonian, so that $\left(-\hbar^{2} \partial_{x}^{2}+e_{0}^{2} B^{2} x^{2}\right) u_{n}(x)=$ $\hbar e_{0} B(2 n+1) u_{n}(x)$.

The equation (1) can be put further, after applying the expansion (4), in the matrix form:

$$
\sum_{n^{\prime}} \mathbf{M}_{n n^{\prime}} \psi_{q}\left(n^{\prime}, k, \omega\right)=\zeta_{q}(n, k, \omega)
$$

where the symmetrical tridiagonal matrix $\mathbf{M}$ has the nonzero elements:

$$
\begin{aligned}
& \mathbf{M}_{00}=i \Gamma_{0}^{-1}\left(\frac{E k}{B}-\omega\right)+\widetilde{a}+\frac{\hbar e_{0} B}{2 m}+\frac{\hbar^{2}(1-\cos q s)}{m_{c} s^{2}} \\
& \mathbf{M}_{n n}=\mathbf{M}_{00}+\frac{\hbar e_{0} B}{m} n ; \\
& \mathbf{M}_{n+1, n}=\mathbf{M}_{n, n+1}=-i \Gamma_{0}^{-1} E \sqrt{\frac{e_{0}}{2 \hbar B}} \sqrt{n+1}
\end{aligned}
$$

and where the new noise terms $\zeta_{q}(n, k, \omega)$, corresponding to the expansion rule (4), are delta-correlated such as $\left\langle\zeta_{q}(n, k, \omega) \zeta_{q^{\prime}}^{*}\left(n^{\prime}, k^{\prime}, \omega^{\prime}\right)\right\rangle=2 \Gamma_{0}^{-1} k_{B} T(2 \pi)^{3} \delta(k-$ $\left.k^{\prime}\right) \delta\left(q-q^{\prime}\right) \delta\left(\omega-\omega^{\prime}\right) \delta_{n n^{\prime}}$.

By solving Eq. (5), and taking into account the expansion form (4), one obtains further the correlation function of the order parameter:

$$
\begin{aligned}
& \left\langle\psi_{l}(x, y, t) \psi_{l}^{*}\left(x^{\prime}, y, t\right)\right\rangle=2 \Gamma_{0}^{-1} k_{B} T \\
& \cdot \int \frac{d k}{2 \pi} \int \frac{d \omega}{2 \pi} \int \frac{d q}{2 \pi} \sum_{n} \sum_{n^{\prime}} u_{n}\left(x+\frac{\hbar k}{e_{0} B}\right) \\
& \cdot u_{n^{\prime}}\left(x^{\prime}+\frac{\hbar k}{e_{0} B}\right)\left(\mathbf{M}^{*} \cdot \mathbf{M}\right)_{n n^{\prime}}^{-1}(q, k, \omega),
\end{aligned}
$$

where the expression $\left(\mathbf{M}^{*} \cdot \mathbf{M}\right)_{n n^{\prime}}^{-1}$ is to be understood as the element of the inverted matrix.

It is hereafter more convenient to rescale the integration variables to the new ones

$$
\omega^{\prime}=\frac{\Gamma_{0}^{-1}}{a_{0}}\left(\omega-\frac{E k}{B}\right) ; \quad q^{\prime}=q s ; \quad k^{\prime}=\frac{\hbar}{e_{0} B} k,
$$

and to introduce the reduced field magnitudes

$$
h=\frac{B}{B_{c 2}(0)}=\frac{\hbar e_{0} B}{2 m a_{0}} \quad \text { and } \quad E^{\prime}=\frac{E e_{0} \xi_{0} \Gamma_{0}^{-1}}{4 \sqrt{3} a_{0} \hbar}=\frac{E}{E_{0}}
$$

where $E_{0}=16 \sqrt{3} k_{B} T / \pi e \xi_{0}$ is defined as in Refs. 6 and 7. One can thus use further instead of the matrix $\mathbf{M}$ the following matrix $\mathbf{M}^{\prime}=a_{0}^{-1} \mathbf{M}$ having no physical dimension, so that

$$
\begin{aligned}
\mathbf{M}_{00}^{\prime} & =-i \omega^{\prime}+\widetilde{\varepsilon}+\frac{r}{2}\left(1-\cos q^{\prime}\right)+h ; \\
\mathbf{M}_{n n}^{\prime} & =\mathbf{M}_{00}^{\prime}+2 h n ; \\
\mathbf{M}_{n+1, n}^{\prime} & =\mathbf{M}_{n, n+1}^{\prime}=-i 2 \sqrt{6} \frac{E^{\prime}}{\sqrt{h}} \sqrt{n+1} \\
r & =2 \hbar^{2} / a_{0} m_{c} s^{2}=\left(2 \xi_{0 c} / s\right)^{2} .
\end{aligned}
$$

After writing Eq. (7) in the newly introduced variables and performing the integral over $k^{\prime}$, we are able to compute the fluctuation Cooper pair density $\left\langle|\psi|^{2}\right\rangle$ and write the self-consistent equation (3) for the renormalized reduced temperature parameter $\widetilde{\varepsilon}$ under the form:

$$
\widetilde{\varepsilon}=\ln \frac{T}{T_{0}}+g T 4 h \int_{-\infty}^{\infty} \frac{d \omega^{\prime}}{2 \pi} \int_{-\pi}^{\pi} \frac{d q^{\prime}}{2 \pi} \sum_{n} \mathbf{Q}_{n n}\left(q^{\prime}, \omega^{\prime}\right)
$$

where we have introduced the hermitian matrix $\mathbf{Q}=\left(\mathbf{M}^{\prime *} \cdot \mathbf{M}^{\prime}\right)^{-1}$ and the parameter $g=$ $2 \mu_{0} \kappa^{2} e^{2} \xi_{0}^{2} k_{B} /\left(\pi \hbar^{2} s\right)$. The expression of the quartic term coefficient ${ }^{11} b=\mu_{0} \kappa^{2} e_{0}^{2} \hbar^{2} / 2 m^{2}$ was also taken into account, with $\kappa$ being the Ginzburg-Landau parameter $\kappa=\lambda_{0} / \xi_{0}$. It must be noticed that the equation for the renormalized reduced temperature, $\widetilde{\varepsilon}$, is highly nonlinear, since the parameter $\widetilde{\varepsilon}$ enters the $\mathbf{M}_{00}^{\prime}$ expression (10) and therefore the $\mathbf{Q}$ matrix elements. 
Analogously, starting from the correlation function (7) written in the new variables from Eqs. (8) and (9), we can eventually find a simpler form for the fluctuation conductivity:

$\sigma=\frac{e^{2}}{4 \hbar s} \frac{2 h^{3 / 2}}{E^{\prime} \sqrt{6}} \int_{-\infty}^{\infty} \frac{d \omega^{\prime}}{2 \pi} \int_{-\pi}^{\pi} \frac{d q^{\prime}}{2 \pi} \sum_{n} \sqrt{n+1} \operatorname{Im}\left(\mathbf{Q}_{n+1, n}\right)$.

where the recursive properties of the $u_{n}(x)$ functions and the $\mathbf{Q}$ matrix hermiticity were also used.

In both expressions Eqs. (11) and (12), the sum over the Landau levels must be cut-off at some maximum index $N_{c}$, reflecting the inherent UV divergence of the Ginzburg-Landau theory. The classicall.14 procedure is to suppress the short wavelength fluctuating modes through a momentum (or, equivalently, kinetic energy) cut-off condition, which, in terms of the Landau level representation writes $11.14\left(\hbar e_{0} B / m\right)\left(n+\frac{1}{2}\right) \leq$ $c a_{0}=c \hbar^{2} / 2 m \xi_{0}^{2}$, where $c$ is an adimensional cut-off parameter of the order of unity. A total energy cut-off was also proposed $\stackrel{15}{\frac{15}{5}}$ which eliminates the most energetic fluctuations and not only those with short wavelengths, and whose physical meaning was recently shown to follow from the uncertainty principle $\underline{\underline{16}}$ which imposes a limit to the confinement of the superconducting wave function. However, in the critical fluctuation region the two cut-off prescriptions almost coincide quantitatively, due to the low reduced-temperature $\varepsilon$ with respect to $c$, so that we shall apply for simplicity the cut-off procedure in its classical form. In terms of the reduced magnetic field $h$, it writes thus $h\left(N_{c}+\frac{1}{2}\right)=c / 2$. In this way, the matrices $\mathbf{M}, \mathbf{M}^{\prime}$ and $\mathbf{Q}$ are truncated at $N_{c}+1$ lines and columns, and the formulae (11) and (12) can be computed numerically.

The renormalization procedure consists thus in using the reduced temperature parameter $\widetilde{\varepsilon}$, renormalized by solving Eq. (11), in the conductivity expression (12). This procedure causes the critical temperature to shift downwards with respect to the bare mean-field transition temperature $T_{0}$. In analogy with the Gaussian fluctuation case, we shall adopt as definition for the critical temperature $T_{c}(E, B)$ the vanishing of the reduced temperature, $\widetilde{\varepsilon}=0$. In practice, one knows experimentally the actual critical temperature $T_{c}(0,0) \equiv T_{c 0}$ measured at very low electrical field and with zero magnetic field, so that one would have to consider Eq. (11) in the zero-fields limit. The relationship between $T_{c 0}$ and $T_{0}$ has been however already found in Ref. 12 and writes $T_{0}=T_{c 0}[\sqrt{c / r}+\sqrt{1+(c / r)}]^{2 g T_{c 0}}$. Now, having $T_{0}$ one can use Eq. (11) for any temperature $T$ and fields $E$ and $B$ in order to find the actual renormalized $\widetilde{\varepsilon}(T, E, B)$, and further the conductivity $\sigma(T, E, B)$ from Eq. (12).

In order to illustrate the main features of our model, we take as example a common material, like the optimally doped $\mathrm{YBa}_{2} \mathrm{Cu}_{3} \mathrm{O}_{6+x}$. Typical characteristic parameters are then: $s=1.17 \mathrm{~nm}$ for the interlayer distance, $\xi_{0}=1.2$ $\mathrm{nm}$ and $\xi_{0 c}=0.14 \mathrm{~nm}$ for the zero-temperature in-plane and out-of-plane coherence lengths, respectively, $\kappa=70$ for the Ginzburg-Landau parameter and $T_{c 0}=92 \mathrm{~K}$ for the critical temperature under very small electric field and no magnetic field. We also choose for convenience a linear temperature extrapolation for the normal state resistivity which vanishes at $T=0$, and has a typical value $\rho_{N}=84 \mu \Omega \mathrm{cm}$ at $T=200 \mathrm{~K}$.

In Fig. 11 the resistivity curves computed according to Eqs. (11) and (12) are shown for different magnitudes of the magnetic field, at a fixed value of a strong electric field. The zero magnetic field curve is however computed within the model presented in Ref. 12, that treats the non-ohmic fluctuation conductivity in arbitrarily strong electric field and zero magnetic field, in the Hartree approximation and with consideration of the UV cut-off. We can notice that the numerical results obtained for finite magnetic fields tend to approach the curve computed according to Ref. 12 while $B$ decreases towards zero.

Figure 10 shows the complementary case of a fixed magnetic field and different magnitudes of the inplane electric field. The fact that the curves approach the result from the UD model in the vanishing electric field limit can be easily proven also analytically. Equation (11) can be directly written in the $E=0$ limit, and since in this case the M-matrix becomes diagonal, the inverted matrix $\mathbf{Q}=\left(\mathbf{M}^{\prime *} \mathbf{M}^{\prime}\right)^{-1}$ can be trivially computed as having the elements $\mathbf{Q}_{n n}^{(0)}=\left\{\omega^{\prime 2}+\left[\widetilde{\varepsilon}+r\left(1-\cos q^{\prime}\right) / 2+h(2 n+1)\right]^{2}\right\}^{-1}$, so that the two integrals over $\omega^{\prime}$ and $q^{\prime}$ can be immediately solved and yield $\left.\widetilde{\varepsilon}\right|_{E=0}=\ln \frac{T}{T_{0}}+g T \cdot 2 h \sum_{n=0}^{N_{c}} D_{n}$, with $D_{n}=[(\widetilde{\varepsilon}+h+2 n h)(\widetilde{\varepsilon}+h+2 n h+r)]^{-1 / 2}$. This is coincident with the analogous equation found by UD for the renormalized reduced temperature in the linear response approximation.

Equation (12) can be also processed in the vanishing electric field limit by expanding the $\mathbf{Q}$-matrix up to the linear term in $E^{\prime}$, so that one obtains $\operatorname{Im} \mathbf{Q}_{n+1, n}^{(1)}=$ $4 \sqrt{6} E^{\prime} \sqrt{h} \sqrt{n+1} \mathbf{Q}_{n n}^{(0)} \mathbf{Q}_{n+1, n+1}^{(0)}$, and after solving the integrals over $\omega^{\prime}$ and $q^{\prime}$, the result becomes $\left.\sigma\right|_{E \rightarrow 0}=$ $\left(e^{2} / 4 \hbar s\right) \sum_{n=0}^{N_{c}}(n+1)\left(D_{n}-2 D_{n+\frac{1}{2}}+D_{n+1}\right)$. This latter expression matches thus the linear response approximation for the in-plane conductivity found by UD, as well as the corresponding formula in the paper by Ikeda et al ${ }^{10}$

Figure 15 shows a further illustration of the effect obtained by the simultaneous application of the magnetic and electric fields, as compared to the individual effects produced by each field alone. The zero-fields curve is computed according to the LD model with the UV cutoff included,, 12.15 namely $\left.\left(16 \hbar s / e^{2}\right) \sigma(\widetilde{\varepsilon})\right|_{E, B=0}=$ $[\widetilde{\varepsilon}(\widetilde{\varepsilon}+r)]^{-1 / 2}-[(\widetilde{\varepsilon}+c)(\widetilde{\varepsilon}+c+r)]^{-1 / 2}-c(c+$ $\widetilde{\varepsilon}+r / 2)[(c+\widetilde{\varepsilon}+r)(c+\widetilde{\varepsilon})]^{-3 / 2}$, where the renormalized reduced temperature parameter $\widetilde{\varepsilon}$ is given by the self-consistent Hartree $\operatorname{approximation}^{12.14} \widetilde{\varepsilon}_{E, B=0}=\ln \left(T / T_{0}\right)+$ 

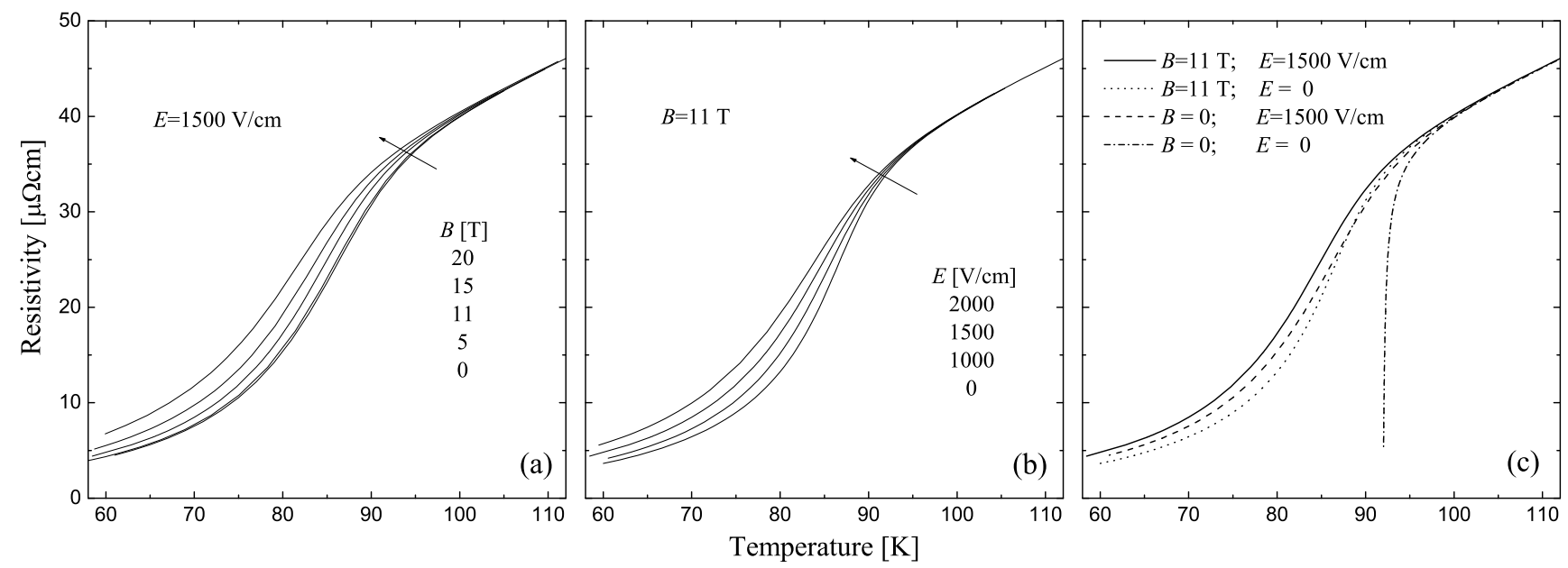

FIG. 1: Resistivity as a function of temperature for different combinations of the perpendicularly applied magnetic field and the in-plane electric field. The material parameters are given in the text. The arrows indicates the increasing direction of the relevant variable field. (a) Fixed electric field and variable magnetic field; the zero magnetic field curve is computed according to the model from Ref. 12. (b) Variable electric field at constant magnetic field; the zero electric field curve (linear response limit) is computed according to the UD model. The UV cut-off parameter $c=1$ in our model corresponds with limiting the sum on the Landau levels at the index $1 / 2 h$ in the UD model. (c) Effects produced by the individually (dash and dot curves) and the simultaneously (solid curve) applied strong electric and magnetic fields; the resistivity curve in the absence of applied fields (dash-dot curve) corresponds to the LD model taken in the Hartree approximation and with considering the UV cut-off.

$2 g T \ln [(\sqrt{\widetilde{\varepsilon}+c}+\sqrt{\widetilde{\varepsilon}+c+r}) /(\sqrt{\widetilde{\varepsilon}}+\sqrt{\widetilde{\varepsilon}+r})]$.

The values for the electric and magnetic fields in Fig. 11: were chosen such that their individual effect be almost similar. One can notice that the simultaneous application of the two fields brings however only a slight enhancement of the superconducting fluctuation suppression. Nevertheless, the non-ohmic behaviour of the fluctuation conductivity at high electric fields still remains quantitatively important for commonly used HTSC also in the presence of strong magnetic fields, so that experimental investigations could be able to discern between the applicability of this model in competition with the linear response approximation.

In summary, we have treated the critical fluctuation conductivity for a layered superconductor in perpendic- ular magnetic field, in the frame of the self-consistent Hartree approximation, for an arbitrary in-plane electric field value. The main results are the formulae (12) for the fluctuation conductivity, and (11) for the renormalized reduced-temperature parameter. In the two limit cases, namely for $(E=0, B>0)$ and for $(E>0, B=0)$, the corresponding solutions were found, analytically and respectively numerically, to reduce to previous results of existing theories. Qualitatively, the simultaneous application of the two fields results in a slight additional suppression of the superconducting fluctuation, compared to the case when the fields are individually applied.

This work was supported by the Austrian Fonds zur Förderung der wissenschaftlichen Forschung.
* Electronic address: ipuica@ap.univie.ac.at

1 L. Aslamazov and A. Larkin, Phys. Lett. 26A, 238 (1968).

${ }^{2}$ W. E. Lawrence and S. Doniach, in Proc. of the 12th Int. Conf. on Low-Temp. Phys., Kyoto, Japan, 1970, edited by E. Kanda (Keigaku, Tokyo, 1971), p. 361.

3 J. P. Hurault, Phys. Rev. 179, 494 (1969).

4 A. Schmid, Phys. Rev. 180, 527 (1969).

5 T. Tsuzuki, Progr. Theoret. Phys. (Kyoto) 43, 286 (1970).

${ }^{6}$ A. Varlamov and L. Reggiani, Phys. Rev. B 45, 1060 (1992).

7 T. Mishonov, A. Posazhennikova, and J. Indekeu, Phys. Rev. B 65, 064519 (2002).

8 K. Maki, J. Low. Temp. Phys. 1, 513 (1969).

9 R. A. Klemm, J. Low. Temp. Phys. 16, 381 (1974).

10 R. Ikeda, T. Ohmi, and T. Tsuneto, J. Phys. Soc. Jap. 60,
1051 (1991), and references therein.

11 S. Ullah and A. T. Dorsey, Phys. Rev. B 44, 262 (1991).

12 I. Puica and W. Lang, Phys. Rev. B 68, 054517 (2003).

13 W. E. Masker, S. Marcelja, and R. D. Parks, Phys. Rev. 188, 745 (1969).

14 T. Mishonov and E. Penev, Int. J. Mod. Phys. B 14, 3831 (2000).

15 C. Carballeira, S. R. Curras, J. Vina, J. A. Veira, M. V. Ramallo, and F. Vidal, Phys. Rev. B 63, 144515 (2001), and references therein.

16 F. Vidal, C. Carballeira, S. R. Currás, J. Mosqueira, M. V. Ramallo, J. A. Veira, and J. Vina, Europhys. Lett. 59, 754 (2002). 